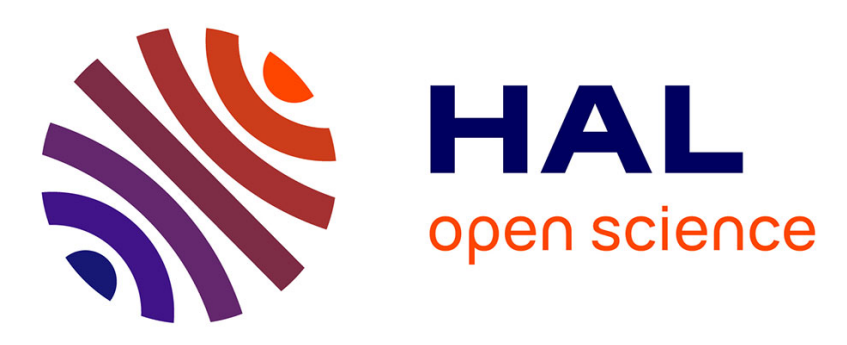

\title{
Mechanical characterization of rubber from one heterogeneous test
}

Thomas Guélon, Evelyne Toussaint, Jean-Benoit Le Cam

\section{To cite this version:}

Thomas Guélon, Evelyne Toussaint, Jean-Benoit Le Cam. Mechanical characterization of rubber from one heterogeneous test. Sixth European Conference on Constitutive Models for Rubber (ECCMR VI), Sep 2009, Dresde, Germany. pp.193-198. hal-01387691

\section{HAL Id: hal-01387691 \\ https://hal.science/hal-01387691}

Submitted on 26 Oct 2016

HAL is a multi-disciplinary open access archive for the deposit and dissemination of scientific research documents, whether they are published or not. The documents may come from teaching and research institutions in France or abroad, or from public or private research centers.
L'archive ouverte pluridisciplinaire HAL, est destinée au dépôt et à la diffusion de documents scientifiques de niveau recherche, publiés ou non, émanant des établissements d'enseignement et de recherche français ou étrangers, des laboratoires publics ou privés. 


\title{
Mechanical characterization of rubber from one heterogeneous test
}

\author{
T. Guélon \\ Laboratoire d'Ingénierie pour les Systèmes Complexes (LISC), Aubière cedex, France
}

\section{E. Toussaint \& J.-B. Le Cam}

Laboratoire de Mécanique et Ingénieries (LaMI) Blaise Pascal University (UBP), French Institute for Advanced Mechanics (IFMA), Campus de Clermont-Ferrand / les Cézeaux, Aubière Cedex, France

\begin{abstract}
The present study deals with the mechanical characterization of elastomeric materials. Classically, three homogeneous tests are considered to identify constitutive parameters, namely Uniaxial Tensile (UT), Pure Shear (PS) and Equibiaxial Tensile (ET) tests. Here, a new method is proposed: it consists in inducing the three previous homogeneous tests from only one heterogeneous mechanical test. For this purpose, a conventional tensile machine is used and a new apparatus is designed to be adapted on the machine. The test-induced heterogeneity is discussed related to two criteria based on the existence of UT, PS and ET and on the distribution of the maximal principal elongation at each material points of the sample surface. Experimentally, kinematic fields are provided on the sample surface by an image correlation code suitable for large deformations. Finally, an inverse technique, so-called Virtual Field Method, is used to identify the material parameters in the framework of the Mooney hyperelasticity.
\end{abstract}

\section{INTRODUCTION}

The behaviour of rubber-like materials is generally modelled in the framework of hyperelasticity. Numerous constitutive relations are available in the literature (Marckmann and Verron 2006). The identification of the material parameters that govern the constitutive equations is still a difficult task. Classically, three homogeneous tests are considered to identify constitutive parameters, namely uniaxial tensile (UT), pure shear (PS) and equibiaxial tensile (ET) (Ward and Hadley 1993), (Sasso et al. 2008) and a trade-off between the different sets of values obtained for each type of test must be found. These tests are based on the strong assumption of homogeneity of the kinematic fields induced by each test.

In the present work, a new approach based on a heterogeneous test that simultaneously generates the three types of strain states abovementioned is pro-posed. For that purpose, the authors propose to design and adapt a new apparatus on a conventional uniaxial tensile machine that generates a heterogeneous strain state. The sample geometry and the loading conditions are defined beforehand by numerical investigations. Two criteria are defined to discuss the heterogeneity induced by the test.
Kinematic fields are provided by a Digital Image Correlation software suitable for large deformations: Correli ${ }^{L M T}$ (Hild 2002). Constitutive parameters are identified using an inverse method called the virtual field method.

\section{CHOICE OF THE SAMPLE GEOMETRY AND THE LOADING CONDITIONS}

In this section, a heterogeneous test that combines UT, PS and ET from a conventional tensile machine is presented. A numerical approach is used to choose the sample geometry and the loading conditions in order to generate sufficient heterogeneity of the kinematic fields. Criteria used to estimate Test-Induced Heterogenity (TIH) are defined and the chosen sample geometry and loading conditions are presented.

\subsection{Test-induced heterogenity}

We analyse the heterogenity induced by the test using the $I_{1}-I_{2}$ diagram where $I_{1}$ and $I_{2}$ are the first and second invariants of the right Cauchy-Green tensor C (Holzapfel 2000). This diagram allow us to determine the loading condition applied at 


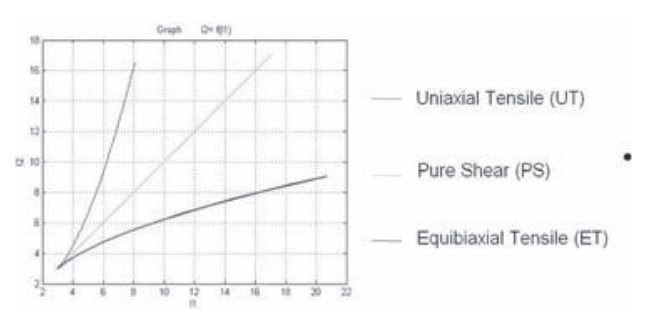

Figure 1. Three basic loading cases.

each material point of the kinematic fields. In this diagram (see Fig. 1), the three curves UT, PS and ET are plotted. The localization of any point in this diagram indicates the corresponding loading condition: it can be only one of the three previous loadings or a combination of two of them.

In order to characterize $\mathrm{TIH}$, we propose two criteria. Firstly, the test has to generate the three homogeneous loading conditions, namely UT, PS and ET. Secondly, for each loading condition, i.e. UT, PS and ET, a distribution of the maximal principal stretch ratio (defined as the ratio between actual and initial lengths) has to be observed and must be sufficiently large considering the chosen constitutive equations and its field of validity. In the following, these criteria are used to choose both sample geometry and loading conditions.

\subsection{Sample geometry and loading conditions}

In the present study, a uniaxial tensile machine is used. In order to generate heterogeneity in the kinelatic fields, a change in geometry (for instance by adding notches or holes) could be considered. However, because of large deformations, the strain state obtain in an elastomer tends to be homogeneous, i.e. TU, whatever the geometry. So, numerical investigations are carried out to choose both sample geometry and biaxial loading conditions that generate a high heterogeneity level. The sample geometry and the loading conditions applied are presented in Figure 2. The sample geometry, which corresponds to a three branch sample, is $2 \mathrm{~mm}$ thick, $60 \mathrm{~mm}$ high and the branches are $20 \mathrm{~mm}$ in width. The bottom branch is clamped, the loading is carried out by applying prescribed displacement following the axis of the two other perpendicular branches.

A Finite Element Analysis is performed using the Ansys 10.0 package to discuss the TIH. Element type plane 182 is used to ensure the incompressibility assumption. The mesh is composed of 640 elements. For the sake of simplicity, we have chosen the Mooney strain energy density (Mooney 1940):

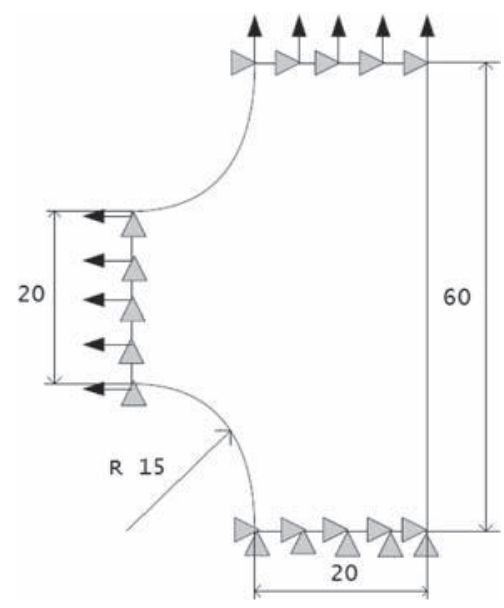

Figure 2. Sample geometry and loading conditions.

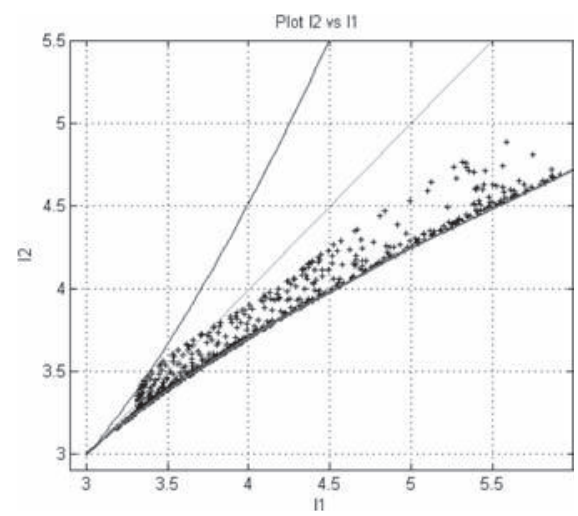

Figure 3. Loading cases in the $\left(I_{1}-I_{2}\right)$ plane.

$W=C_{1}\left(I_{1}-3\right)+C_{2}\left(I_{2}-3\right)$

where $C_{1}$ and $C_{2}$ are the material parameters. Their values are chosen equal to $0.4 \mathrm{MPa}$ and $0.04 \mathrm{MPa}$, respectively, which are typical values for carbon black filled natural rubber.

Figure 3 shows that in the $\left(I_{1}-I_{2}\right)$ diagram, the three homogeneous tests are satisfactorily represented and distributed. The corresponding maximum principal elongation (see Fig. 4) of each loading case at the sample surface is deduced from Figure 3.

Global stretch ratios (defined as the ratio between actual and initial sample lengths) of 1.71 and 1.42 are prescribed following the horizontal and the vertical axes, respectively. The equivalent maximum stretch ratio is higher for UT than 


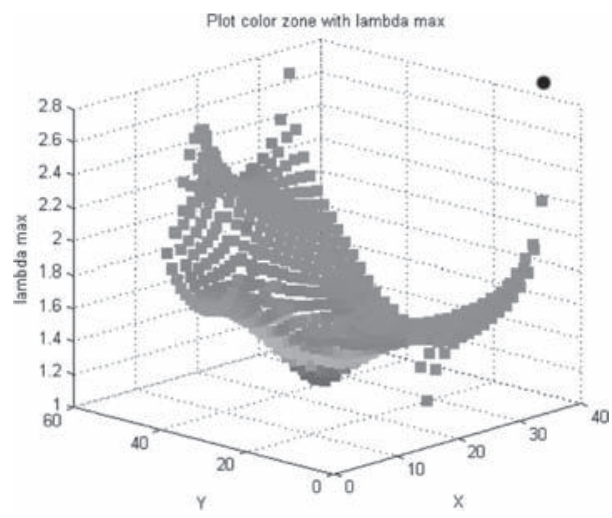

Figure 4. Numerical TIH visualization: maximum value of the principal stretch ratio.

for PS and ET. This is explained by the fact that PS and ET are obtained by the extension of the branches. From Figure 4, one can conclude that each loading case is satisfactorily represented in terms of distribution of the maximum principal stretch ratio.

\section{EXPERIMENTAL SET-UP}

The numerical configuration is now used to perform the test with a conventional uniaxial testing machine. To prescribe a biaxial loading condition, a new apparatus is designed. It is presented in the first part of this section. Then, the measurement of the displacement fields is precisely described.

\subsection{New apparatus}

The testing machine, referred to as MTS 858 Elastomer Test System, has a loading capacity of $15 \mathrm{kN}$. The loading cell is $1 \mathrm{kN}$ capacity. A new tensile apparatus is designed to be adapted to the uniaxial tensile machine. It allows to generate ET at the sample centre. This apparatus is mounted on the left testing machine column (see Fig. 5).

Figure 6 presents a picture of the apparatus. It is composed of four parts:

- part A allows the apparatus to be fixed on one of the two columns;

- part B is a grip in which the horizontal branch of the sample is fixed;

- part C corresponds to a helical slide that ensures the horizontal branch of the sample to be stretched;

- part D is a ruler used to measure the horizontal displacement of the grip.

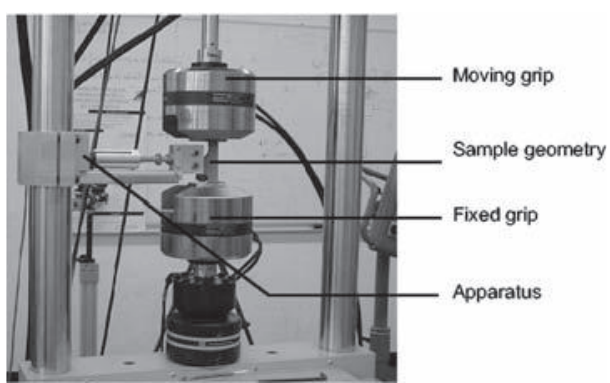

Figure 5. Experimental set-up.

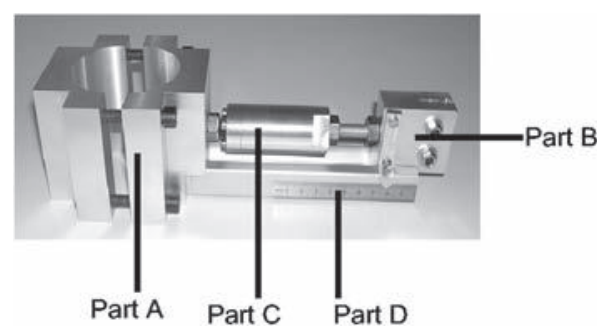

Figure 6. Proposed apparatus.

In practise, the two vertical branches of the sample are fastened on the grips of the conventional testing machine, then the branch perpendicular to the previous ones is fastened on the apparatus grip.

\subsection{Measurement of the kinematic fields}

Digital Image Correlation (DIC) technique is used to measure the displacement fields at the sample surface. For that purpose, images are shot with a cooled 12-bit dynamic CCD camera with $1376 \times 1040$ squared pixels and CORRELI ${ }^{L M T}$ software is used. The DIC technique is well-suited for measuring large strains and has already been used in the case of elastomeric materials (Chevalier et al. 2001). To determine the displacement field of a given image with respect to a reference image, one considers a set of sub-images (i.e., a square region that contains $\mathrm{N} \times \mathrm{N}$ pixels). This set is referred to as Zone of Interest (ZOI). A suitable correlation function is used to calculate the displacement of the centre of a given ZOI in two images captured at different stages of an experiment. In the present work, special attention is paid to the choice of the size of the ZOIs that define the region of interest (ROI). Here, the size of the ZOI is equal to 16 pixels with a shift of 16 pixels. This parameter characterises the measurement grid. To improve the image contrast, white paint is sprayed on the sample surface before testing. 


\section{IDENTIFICATION OF THE MATERIAL PARAMETERS}

In this section, we present the method used to identify the material parameters of a given model from one heterogeneous test. It must be emphasized that no closed-form solution generally exists for such a problem, thereby meaning that no simple relation between local measurements, load, specimen geometry and unknown parameters is available. Extracting constitutive parameters in this case is a major issue which must be tackled using relevant tools. We propose here to use the so-called Virtual Fields Method (VFM) which has recently be used for characterizing elastomeric materials within the framework of large deformations (Promma et al. 2009).

The VFM relies on the Principle of Virtual Work (PVW) which can be regarded as the global equilibrium of the specimen under study or the weak form of the local equations of equilibrium (Dym and Shames 1973). In case of large deformations, assuming a plane state of stress and neglecting body forces such as weight, the PVW can be written as follows:

$$
\int_{S_{0}} \Pi: \frac{\partial U^{*}}{\partial X} d S_{0}+\int_{\partial S_{0}}(\Pi . n .) . U^{*} d l=0 \forall U^{*} K . A .
$$

where $\Pi$ is the first Piola-Kirchhoff stress tensor (PK1), the $X$ s are the Lagrangian coordinates, $U^{*}$ is a kinematically admissible virtual field, $S_{0}$ is the surface of the specimen in the initial configuration the boundary of $S_{0}$ and $n$ the unit vector perpendicular to this boundary. Considering the Mooney strain energy density, one can express the stress components as functions of the actual strain components. This leads to the following equation:

$$
\begin{aligned}
C_{1} \int_{S_{0}} A & : \frac{\partial U^{*}}{\partial X} d S_{0}+C_{2} \int_{S_{0}} B: \frac{\partial U^{*}}{\partial X} d S_{0} \\
& =\int_{\partial S_{0}}(\Pi . n .) . U^{*} d l=0 \forall U^{*} K . A .
\end{aligned}
$$

where $\mathrm{A}$ and $\mathrm{B}$ are quantities depending on the components of the deformation gradient tensor $F$. This equation is linear with respect to the unknown parameters $C_{1}$ and $C_{2}$. In the integrands above, there are two types of terms: $\mathrm{A}$ and $\mathrm{B}$ on one hand, $\partial U^{*} / \partial X$ on the other hand. $\mathrm{A}$ and $\mathrm{B}$ are directly deduced from the full-field measurements whereas $\partial U^{*} / \partial X$ is derived from the virtual field $U^{*}$. At this stage, the idea consists in writing the PVW with two different virtual fields $U^{*}$. Since actual strain fields are heterogeneous, this leads to two independent equations where $C_{1}$ and $C_{2}$

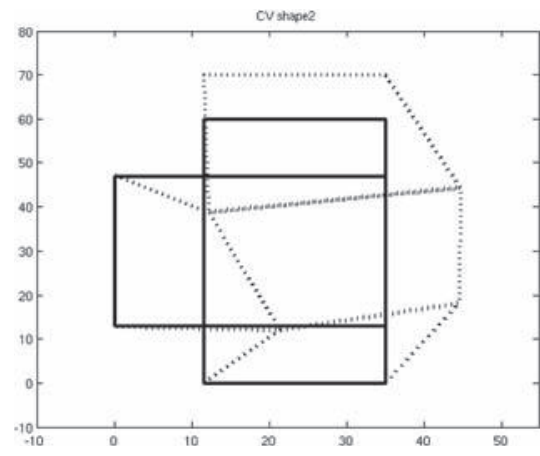

Figure 7. Example of one optimized virtual fields (dotted line) used for identifying $C_{1}$ and $C_{2}$. The maximum global stretch ratios is superimposed with the virtual mesh (solid line).

are unknown. They are obtained by inverting the linear system.

Choosing at best the two virtual fields is a key issue in the method. In the present case of large deformations and hyperelasticity, it has been decided to use the following heuristic method. A large number of virtual fields is first randomly generated. Then, the set of two virtual fields that leads to the best conditioning of the linear system (assessed with the condition number) is finally chosen for identification purposes. Those two virtual fields provide parameters less sensitive to noisy data. In the present work, virtual fields have been defined piecewise (Toussaint et al. 2006) because of the particular shape of the specimen. Four subregions are used to mesh the specimen because of its particular shape. The virtual displacement defined in each of the four sub-regions is described by polynomial shape functions multiplied by the virtual displacement of the nodes defining the corners of the sub-regions. These shape functions are similar to those employed in the finite element method. Figure 7 presents an example of optimized initial fields used for identifying $C_{1}$ and $C_{2}$ for the maximum global stretch ratios.

\section{RESULTS}

\subsection{Experimental kinematic fields}

The biaxial tensile test is carried out by prescribing a $25 \mathrm{~mm}$ displacement along both the $\mathrm{x}-$ and $y$ - directions shown in Figure 2. The corresponding global stretch ratios are 1.71 and 1.42 along the $\mathrm{x}$ - and $\mathrm{y}$-directions, respectively. In order to avoid the well-known stress accommodation (Mullins 1948; Meunier et al. 2008; Godin et al. 2009) 
over the first mechanical cycles, three cycles are first carried out with the same maximum stretch ratio, thereby partially stabilizing the mechanical response of the specimen. Images are stored for every $1 \mathrm{~mm}$ of prescribed displacement in both directions. The material considered here is a carbon black filled natural rubber. Its composition and some of its mechanical properties are given in Table 1.

A typical view of the horizontal and vertical displacement fields obtained for the maximum prescribed global stretch ratios is shown in Figure 8.

Differentiating these fields provides the four components of the displacement gradient. They are obtained by using a centered finite differences scheme. In the present case, 740 experimental points are processed. This number is close to the number of elements used for numerical simulations and makes it easier to compare numerical and experimental TIH visualizations (see Figs. 3 and 9).

The states of strain of the three homogeneous tests (UT, PS and ET) are represented and the equivalent maximum stretch ratio is higher for UT than for PS and ET. Figure 10 presents the maximum principal stretch ratio for each ZOI.

Here, each loading case is satisfactorily represented in terms of maximum stretch ratio distribution. With regard to the previous analysis and numerical prediction, the TIH satisfies the two criteria used to define a sufficient level of TIH. Some points are encircled in Figures 9 and 10. They correspond to points taht are located at the border of the sample i.e. to zones for which there are insufficient measurement points to obtain an efficient image correlation. To summarize, these experimental results are in good agreement with the numerical prediction in terms of TIH.

Table 1. Material formulation (parts per hundred rubber) and mechanical properties.

\begin{tabular}{lc}
\hline Components & NR \\
\hline Rubber & 100 \\
Zinc oxide & 9.85 \\
Oil & 3 \\
Carbon black & 34 \\
Sulfur & 3 \\
Stearic acid & 3 \\
Antioxidant & 2 \\
Accelerators & 4 \\
\hline Density & 1.13 \\
Shore A hardness & 58 \\
Stress at break (MPa) & 22.9 \\
Elongation at failure & 635 \\
\hline
\end{tabular}
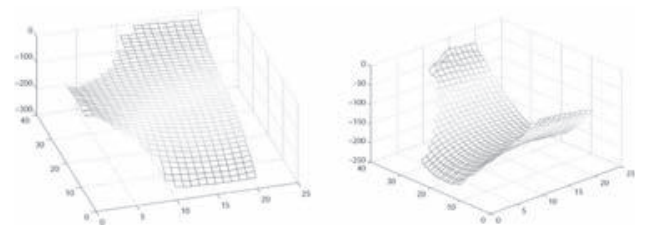

Figure 8. Displacement fields.

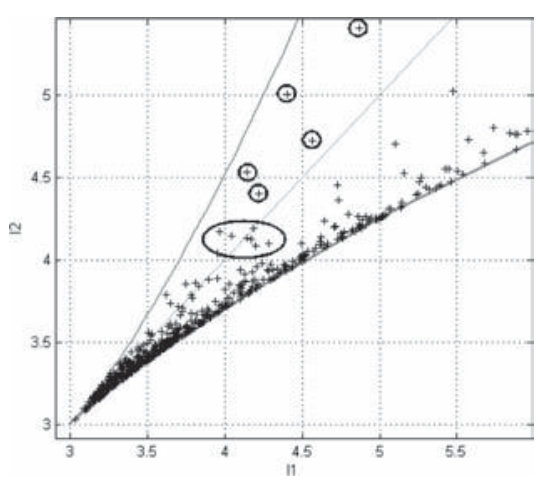

Figure 9. Loading cases in the $\left(I_{1}-I_{2}\right)$ plane.

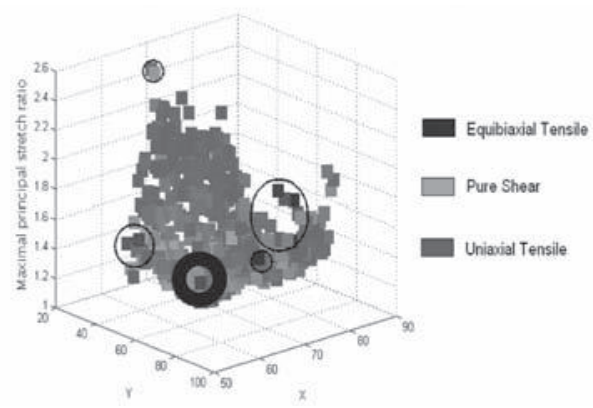

Figure 10. Experimental TIH visualization: maximum value of the principal stretch ratio.

\subsection{Identification results}

Identification results are presented in Figure 11 for each step of the loading. This step is equal to $1 \mathrm{~mm}$ along both the x- and y- directions. $C_{1}$ and $C_{2}$ are calculated at each loading step. The global stretch ratio reported along the horizontal axis in Figure 11 is that prescribed along the y- direction.

Apart from stretch ratios inferior to 1.05 , both $C_{1}$ and $C_{2}$ remain approximately constant while the global stretch ratio increases, thereby showing that the Mooney strain energy density presently used correctly describes the actual mechanical response 

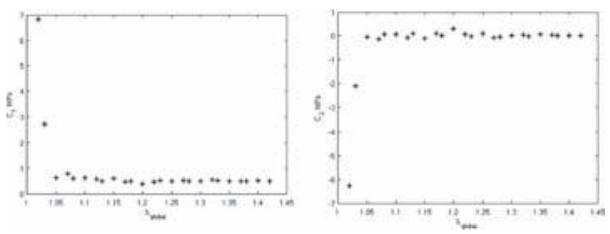

Figure 11. Identification of the material parameters $C_{1}$ and $C_{2}$.

of the specimen. The mean values of $C_{1}$ and $C_{2}$ deduced from these curves are $0.509 \mathrm{MPa}$ and $0.012 \mathrm{MPa}$, respectively. These values are acceptable with regard to thermodynamic considerations (Ball 1977). It should be noted that only the loading steps corresponding to a global stretch ratio along the $y$ - direction ranging between 1.05 and 1.42 are considered here. In fact, identifying $C_{1}$ and $\mathrm{C}_{2}$ in the domain of low stretch ratio leads to unreliable results. This is explained by the fact that the material parameters are not sufficiently activated in this domain. It should be noted that this observation is valid whatever the law considered.

\section{CONCLUSION}

The aim of the present paper is to propose an alternative to the classical method of identifying constitutive parameters of rubber. For that purpose, only one heterogeneous test is performed. Sample geometry and loading conditions are chosen using numerical simulations in order to involve UT, PS and ET at the sample surface. The test-induced heterogeneity is discussed related to two criteria. To perform the heterogeneous test, a new apparatus is designed and is adapted on a conventional tensile machine. Displacement fields are measured on the specimen surface using the DIC technique.

Unknown constitutive parameters are then deduced from these fields using a suitable identification procedure: the Virtual Fields Method extended to hyperelasticity. Results obtained are in agreement with theoretical and numerical expectations, thus confirming the feasibility of the present approach. An interesting perspective would be to identify parameters governing more complicated constitutive models. For instance, it could be interesting to take into account the significant change in volume observed under extension in such a material (Le Cam and Toussaint 2008).

\section{REFERENCES}

Ball, J. (1977). Convexity conditions and existence theorems in nonlinear elasticity. Arch. for Ration. Mech. An. 63, 337-403.

Chevalier, L., S. Calloch, F. Hild, and Y. Marco (2001). Digital image correlation used to analyze the multiaxial behavior of rubber-like materials. Eur. J of Mech. A-Solid 20(2), 169-187.

Dym, C.L. and I.H. Shames (1973). Solid Mechanics A Variational Approach. McGraw-Hill Book Company.

Godin, N., S. Chaki, J. Courbon, S. Deschanel, S. Gillet, and B. Gautier (2009). Acoustic emission potentialities for characterization of mullins effect in natural rubber materials filled with carbon black. Polymer Testing 28, 103-105.

Hild, F. (2002). Correlilmt: a software for displacement field measurements by digital image correlation. Technical Report 254, LMT. Internal report.

Holzapfel, G.A. (2000). Nonlinear Solid Mechanics. $A$ continuum approach for engineering. J. Wiley and Sons.

Le Cam, J.-B. and E. Toussaint (2008). Volume variation in stretched natural rubber: competition between cavitation and stress-induced crystallization. Macromolecules 41(20), 7579-7583.

Marckmann, G. and E. Verron (2006). Comparison of hyperelastic models for rubberlike materials. Rubber Chem. Technol. 79, 835-858.

Meunier, L., G. Chagnon, D. Favier, L. Orgéas, and P. Vacher (2008). Mechanical experimental characterization and numerical modelling of an unfilled silicone rubber. Polymer Testing 27, 765-777.

Mooney, M. (1940). A theory of large elastic deformation. J. Appl. Phys. 11, 582-592.

Mullins, L. (1948). Effect of stretching on the properties of rubber. Rubber Chem. Technol. 21, 281-300.

Promma, N., B. Raka, M. Grédiac, E. Toussaint, J.-B. Le Cam, X. Balandraud, and F. Hild (2009). Application of the virtual fields method to mechanical characterization of elastomeric materials. Int. J. Solids Struct. 46, 698-715.

Sasso, M., G. Palmieri, G. Chiappini, and D. Amodio (2008). Characterization of hyperelastic rubber-like materials by biaxial and uniaxial stretching tests based on optical methods. Polymer Testing 27, 999-1004.

Toussaint, E., M. Grédiac, and F. Pierron (2006). The virtual fields method with piecewise virtual fields. Int. J Mech. Sci. 48, 256-264.

Ward, I.M. and D.W. Hadley (1993). An Introduction to the Mechanical Properties of Solid Polymers. John Wiley and Sons Ltd. 